\title{
INDUSTRY 4.0: A THEORETICAL CONTRIBUTION TO THE CURRENT SCENARIO OF TECHNOLOGY IN BRAZIL
}

\author{
Sangela Azevedo da Silva ${ }^{1}$, Renan de Souza Vasconcelos ${ }^{2}$, Paola Souto Campos ${ }^{3}$
}

\author{
${ }^{1,2,3}$ Centro Universitario do Norte - UNINORTE - Laureate Universites, Brasil, Rua Leonardo Malcher, 715. Centro, \\ Manaus/AM.
}

Email: sangelaazevedo16@ hotmail.com, renandesouzavasconcelos@yahoo.com.br, pscampos@gmail.com

Received: May 23 ${ }^{\text {th }}, 2019$

Accepted: May 30th, 2019

Published: September $30^{\text {th }}$, 2019

Copyright @2016 by authors and Institute of Technology Galileo of Amazon (ITEGAM). This work is licensed under the Creative Commons Attribution International License (CC BY 4.0). https://creativecommons.org/lice nses/by/4.0/

\section{(i) Opea Acteses}

\begin{abstract}
Industrial automation brings a new generation to industry development 4.0. A fourth generation of industrial boosters, machines, processes, and people connect as a collaborative community. In Brazilian industries, there is a technology deployment process 4.0, but at what level of development? The purpose of this article is to promote a discussion among authors of various papers on the magnification of industry 4.0 nationally. How is the work methodology, adopting the development of a bibliographical revision, with the use of published, printed and printed books, in order to explore the context and work areas of the industry 4.0. As a result, one analyzes several concepts, exposes the challenges and discusses two industrial forms.
\end{abstract}

Keywords: Industry 4.0; technology; national.

\section{INDÚSTRIA 4.0: UM APORTE TEÓRICO SOBRE O CENÁRIO ATUAL DA TECNOLOGIA NO BRASIL}

\begin{abstract}
RESUMO
A automação industrial presencia uma nova geração com o desenvolvimento da indústria 4.0. A quarta geração industrial permitirá que sensores, máquinas, processos e pessoas se conectem como uma comunidade colaborativa. Nas indústrias brasileiras, há um processo de implantação da tecnologia 4.0, mas em que nível de desenvolvimento? O objetivo deste artigo é promover uma discussão entre pensamento de vários autores sobre a ampliação da indústria 4.0 a nível nacional. Como metodologia de trabalho, adotou-se o desenvolvimento de uma revisão bibliográfica, baseada em livros, periódicos e impressos diversos, com o intuito de explorar o contexto e cenários criados pela indústria 4.0. Como resultado, tem-se a análise de vários conceitos, expõe-se desafios e discute-se a interação entre a ideia de diversos autores sobre a quarta geração industrial.
\end{abstract}

Palavras-chave: Industria 4.0; tecnologia; nacional.

\section{INTRODUÇÃO}

O termo Indústria 4.0 surgiu na Alemanha em meados de 2011, vinda de um projeto do governo que tinha por objetivo afirmar que o uso de tecnologias era um excelente aliado para a revolução das indústrias de todo o mundo [1]. Com a integração das tecnologias existentes e uma integração entre os processos da produção, era possível obter um melhor resultado do que era conseguido antes, e com isso surgiu os sistemas de produção inteligente. Os resultados obtidos pelas empresas após o uso de tecnologia e integração entre as partes foram muito bem recebidos pelas empresas já que gerou ótimos resultados.

De acordo com [2], as indústrias estão no fim da Terceira Revolução Industrial e caminhando em direção para a Quarta Revolução Industrial. A Figura 1 ilustra a Revolução Industrial no mundo. 


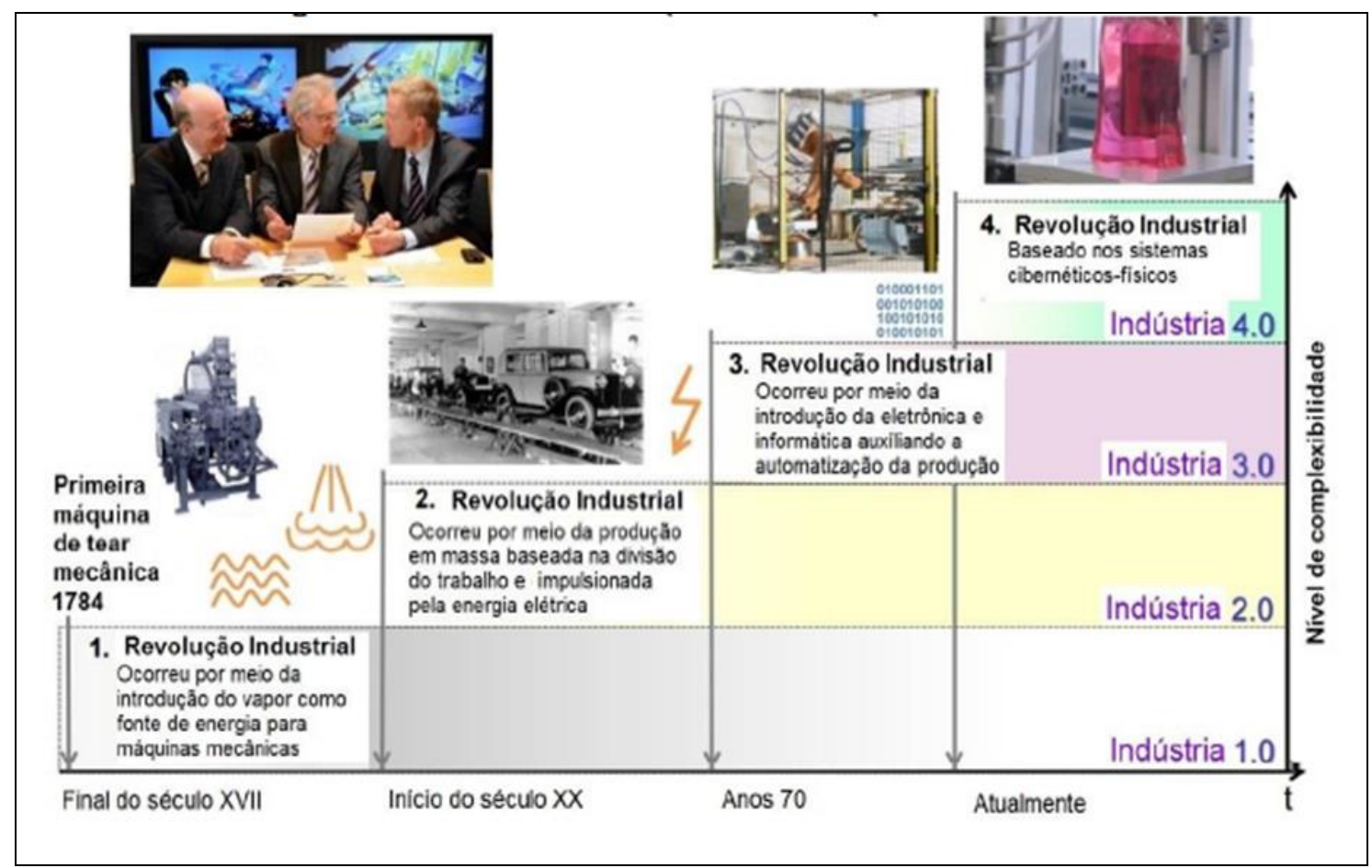

Figura 1. Evolução Industrial. Fonte: [2].

A Indústria 4.0 representa o conjunto de inovações capazes de alavancar a eficiência das operações de forma consistente, auxiliando as organizações em seus diferenciais de competitividade, no futuro próximo. Ao introduzir sistemas inteligentes que permitam a integração entre equipamentos na manufatura e serviços logísticos, a complexidade operacional será naturalmente reduzida, aumentando a robustez e segurança dos processos, bem como a redução dos custos de não qualidade [3].

Dessa forma, apoiada em seus pilares que são: Internet das Coisas, Sistemas Cyber-Físicos, Big Data, Segurança dos Dados, Realidade Aumentada, Robôs Autônomos, Simulação, Manufatura Aditiva, Nuvem e Integração Horizontal e Vertical de Sistemas, a quarta geração da indústria tem como objetivo conectar, digitalmente, fornecedores, equipamentos e as próprias unidades fabris, criando com isso uma cadeia de valor competitiva e integrada.

Acredita-se que o esse sistema atual, focado em novas tecnologias, funcionará diante da condição de um sistema de produção adequado, que integre a inovação ao atual conceito Lean (produção enxuta). Tal discussão da Indústria 4.0 se dá fundamentalmente nos campos da inovação, mas, sobretudo, o que se refere a produtividade, e a implementação efetiva da tecnologia nas fases do processo produtivo.

Um estudo publicado pela Confederação Nacional da Indústria (CNI) em 2016 listou dez tecnologias digitais às empresas e, ao serem questionadas sobre a utilização, $48 \%$ informaram que utilizavam pelo menos uma delas, $15 \%$ não utilizavam nenhuma delas e 6\% desconheciam o assunto (do total, 25\% não responderam à pesquisa).

Diante do exposto, nota-se que há uma forte dificuldade quanto a implementação das tecnologias digitais, o que pode está diretamente atrelada ao alto custo da implantação, e a falta de estudo de retorno de investimento mais claros para os empresários [4].

No médio e longo prazo, para que a indústria brasileira consiga competitividade será essencial a incorporação dessas novas tecnologias, e assim, melhorar a sua participação no mercado global. Além de buscar essa incorporação e o desenvolvimento dessa tecnologia, é preciso uma certa agilidade, a fim de evitar que o gap de competitividade do Brasil em relação aos outros países aumente [5].

É sob a análise dos desafios da implantação da indústria 4.0 no Brasil e seus efeitos econômicos, que o objetivo deste trabalho está pautado.

\section{MATERIAIS E MÉTODOS}

Atrelado ao objetivo proposto, este artigo tem como natureza, uma abordagem qualitativa, um aprofundamento bibliográfico sobre a Indústria 4.0, as tecnologias desenvolvidas pela Indústria 4.0 e as dificuldades de implantar o conceito de Indústria 4.0 no Brasil e como são utilizadas. Assim como uma análise sobre as dificuldades de implementação deste novo conceito que está começando a aparecer nos novos modelos de gestão dos negócios em termos de ganhos econômicos

Uma revisão de literatura de caráter descritiva sobre este conceito foi realizada para a produção deste artigo. Em relação aos procedimentos técnicos, realizou-se uma pesquisa bibliográfica, ao qual foram buscados referenciais teóricos em livros, artigos, sites, revistas, entre outros. Por ser um tema atual e ainda pouco explorado no mundo acadêmico, há um número baixo de conteúdo que possa preencher uma pesquisa extensa. Assim, foram realizadas consultas de referenciais teóricos de 2005 a 2018.

O procedimento de coleta de dados consistiu no levantamento de artigos relevantes dentro da literatura sobre a Quarta Revolução Industrial.

\section{RESULTADOS E DISCUSSÕES}

Segundo [6], a essência da indústria 4.0 está baseada nos sistemas cibernético-físico (CPS) e Internet das Coisas (IoT), que levarão as fábricas a atingirem um novo patamar de produção. $\mathrm{O}$ CPS baseia-se na configuração dinâmica da manufatura. O CPS é a nova geração que integra computadores e a natureza física. Onde, 
a natureza cibernética é um somatório de algoritmos da lógica das unidades de sensores, e a natureza física é a soma das unidades atuadoras com habilidade de interagir e expandir capacidades do mundo físico usando auxílio computacional, tecnologias da comunicação e controle de mecanismos.

A conectividade das "coisas" é de extrema importância, e necessita estar disponível, para que os benefícios consolidados da indústria 4.0 possam ser usufruídos de forma a trazer retorno no tempo previsto. O Governo Federal criou a Câmara de Gestão M2M em 2014 com o objetivo de acompanhar, subsidiar e promover a comunicação M2M no Brasil e possui nove verticais estratégicas: cidades inteligentes, agronegócio, saúde, educação, produtividade industrial, logística, transporte, energia e outras.
Essas verticais irão promover a discussão pública de questões tributárias, geração de oferta, geração de demanda, regulatório, educação, pesquisa e desenvolvimento, segurança e privacidade das informações, interoperabilidade e arranjos produtivos referentes a IoT.

O conceito básico da internet industrial das coisas (IIoT), para processos de manufatura, é a presença generalizada ao nosso redor de sensores, atuadores, dispositivos de identificação por radiofrequência (RFID), etiquetas, códigos, celulares, tablets, etc. Onde, por meio de endereçamento exclusivo as "coisas" possuem capacidade de interagir e cooperar dentro de uma rede para atingir objetivos em comum [7]. A Figura 2 mostra como a IoT entregará soluções inovadoras.

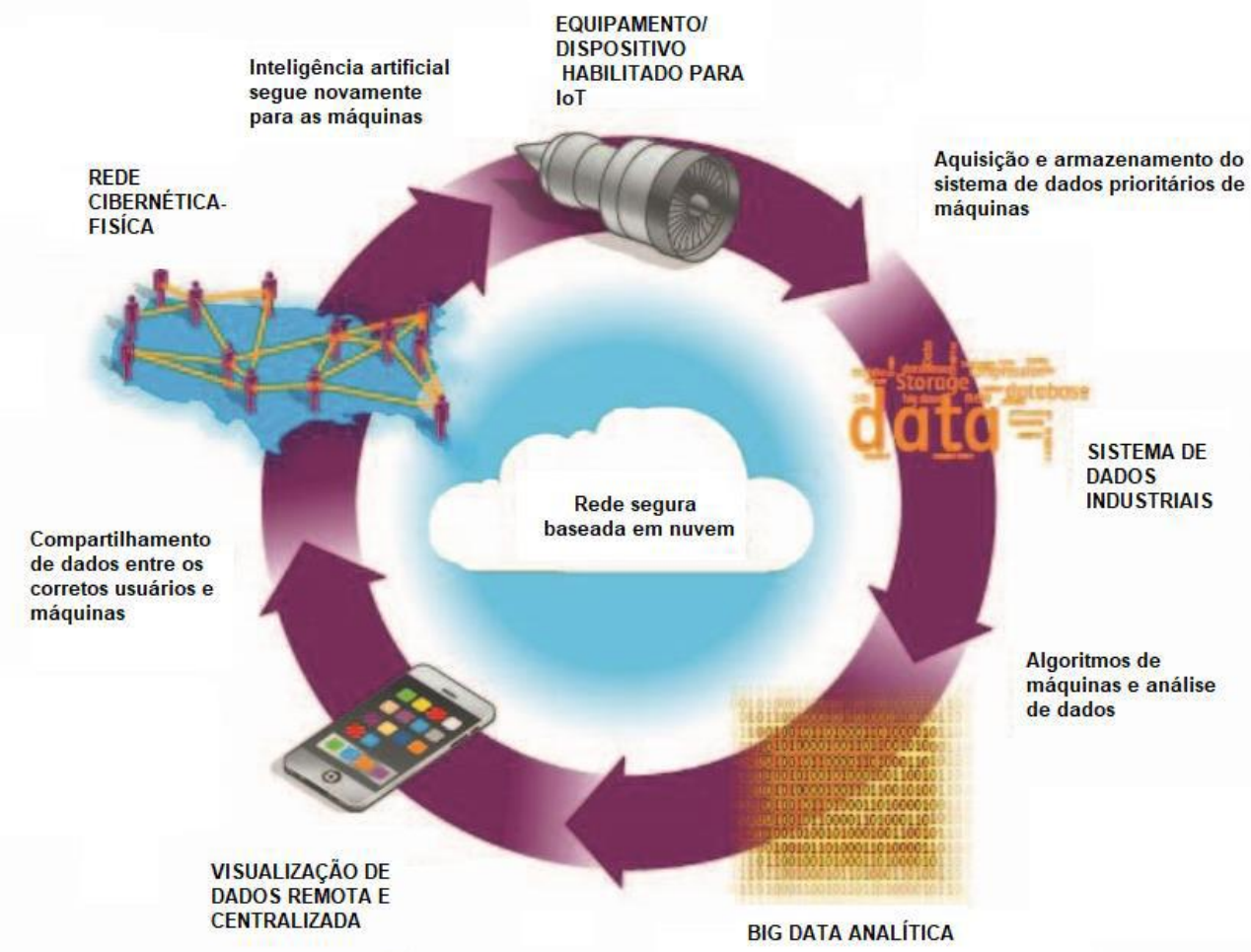

Figura 2. Exemplo de aplicação da IoT. Fonte: [7].

De acordo com a divisão de pesquisas e projetos no campo da internet das coisas. "Coisas" são os participantes ativos de negócios, informações pareadas e processos, conectados para interagir e comunicar entre eles e com o ambiente externo por meio da troca de dados e informações. Diferente dos métodos tradicionais de produção, a configuração dinâmica está acima da produção e de processos envolvidos. Pois o dinamismo torna o sistema capaz de alterar o projeto inicial do produto a qualquer momento.

As conexões entre objetos físicos e o mundo virtual, hardwares cada dia mais sofisticados, além da diversificação progressiva de produtos; geram profundos impactos na área de industrial. Pois, marcam o início da mudança de direção para elementos interconectados. Em um ambiente industrial onde estejam presentes a big data e IoT; as máquinas, dispositivos e produtos podem tomar decisões autonomamente e se auto organizarem dentro de uma rede dinâmica [8].

Diante desses conceitos sobre a essência da indústria 4.0 considera-se que a mesma vai além da simples digitalização, uma forma complexa de inovação com base em diversas tecnologias, ao qual, fará as empresas repensarem como gerir seus negócios e processos, no desenvolvimento de novos produtos e como posicionar no mercado.

Para [9], esse pacote tecnológico a ser implantado pela quarta geração industrial permitirá que as empresas possam revolucionar e aperfeiçoar a gestão do processo produtivo da indústria. O panorama da indústria atual é caracterizado pelo aumento da digitalização, cadeia de valor, modelos de negócios, produção inteligente (Smart Production), processos e produtos.

A indústria 4.0 é um caminho sem volta que garante a competitividade para o futuro. A empresa que não aderir aos seus conceitos, não será capaz de ser competitiva futuramente. Esta tecnologia permitirá tomar decisões extremamente rápidas, assertivas, detectar problemas, evitar falhas e reduzir custos [10].

\section{CONCLUSÕES}

Através deste trabalho foi possível analisar os conceitos de IoT e CPS, que são exemplos de tecnologias da comunicação e informação da indústria 4.0. Essas tecnologias permitem a conexão de robôs e sistemas que integram a produção, e com isso foi avaliada a importância da mão de obra nas indústrias que irão implementar tal tecnologia. 
Foi importante perceber que mesmo a distância de se enxergar a quarta revolução industrial nas indústrias brasileiras, é importante explorar o futuro tecnológico e entender como o mesmo irá afetar a sociedade a partir do momento que revoluciona o processo industrial e agrega ainda mais valor ao produto final.

A tecnologia da indústria 4.0 abre um amplo espaço para o desenvolvimento de melhorias, o que vem dar retorno financeiro as indústrias, e vem com isso fortalecer a economia nacional com redução de energia para se produzir e de manutenção dos equipamentos, tornando o preço do produto ainda mais atraente para o mercado exportador.

Mesmo com um panorama e uma perspectiva baixa de implantação, é necessário entender os conceitos e vantagens da indústria 4.0, pois o Brasil é um país que pode se expor a essas tecnologias, seja pelo aumento da concorrência, seja pelo estabelecimento frequente de parcerias com empresas mais avançadas tecnologicamente.

Automática (ABS) e do Programa de Estabilidade Eletrônica (EPS), e essa melhoria foi resultante da implantação de linhas inteligentes conectadas.

É possível verificar que além da redução de erro humano, a indústria 4.0 pode trazer benefícios financeiros e otimização de recursos quando aumenta a eficiência de todos os processos. A tecnologia está disponível em todos os setores, basta saber identificá-la e usá-la de forma a trazer retorno.

Segundo uma pesquisa da [5], para que seja acelerado o processo de implantação dessas tecnologias no Brasil, o governo deverá focar em investir na infraestrutura digital, promover linhas de financiamento para o mercado e incentivar a educação através de treinamentos, para que seja promovido o conhecimento e estimular os profissionais neste novo cenário de trabalho. Com base nessas considerações acredita-se que há um desencontro entre o esforço das indústrias em se tornarem estruturalmente preparadas para receber a quarta revolução industrial e os programas governamentais de incentivo as universidades e/ou institutos tecnológicos em preparar mão de obra qualificada que agregue valor ao programa.

Consultorias têm estimado os impactos da digitalização na economia e competitividade do país. Segundo a [14], estima que a implementação ligada à Internet das Coisas (Internet of Things) nos mais diversos setores da economia deverão impactar o PIB brasileiro em aproximadamente US\$ 39 bilhões até 2030. Poderá ter um ganho de US\$ 210 bilhões, caso o país crie condições que possam acelerar a implantação das tecnologias relacionadas. Conforme a [15] há uma estimativa de que até 2025 os processos relacionados à Indústria 4.0 reduzirão os custos de manutenção de equipamentos entre $10 \%$ e $40 \%$, reduzir o consumo de energia entre $10 \%$ e $20 \%$ e aumentar a eficiência do trabalho entre $10 \%$ e $25 \%$.

No país já se iniciou os primeiros passos da Indústria 4.0, através da criação da Associação Brasileira de Internet Industrial (ABII). Segundo [13] o objetivo inicial é divulgar a Internet Industrial e fortalecer o cenário no país, através da criação de fóruns de discussão do tema, intercâmbio tecnológico e de negócios, visando o desenvolvimento econômico e a criação de empregos no país.

Diante do exposto acima, tem-se que a indústria 4.0 levará as fábricas a atingirem um novo estilo de produção: mais competitivo, dinâmico e confiável. Consequentemente, o produto terá um menor custo de fabricação e tornará a empresa mais competitiva e economicamente estável frente ao mercado internacional.
Os autores citados mostram grandes ganhos econômicos e as vantagens da tecnologia 4.0, assim como a estabilidade que a tecnologia traz ao processo produtivo.

Porém, no Brasil, as dificuldades de implantação da indústria 4.0 recaem em dificuldades crônicas, que o governo junto as instituições tecnológicas não conseguem solucionar de forma rápida, a acompanhar a evolução das indústrias nos países de primeiro mundo.

\section{REFERÊNCIAS}

[1] Brettel, M.; Friederichsen, N.; Keller, M.; Rosenberg, M. How virtualization, decentralization and network building change the manufacturing landscape: An Industry 4.0 Perspective. International Journal of Mechanical, Industrial Science and Engineering, v. 8, n.1, p. 37-44, 2014. Disponível em: < http://waset.org/publications/9997144>. Acesso em: 09/03/2018.

[2] Kagermann H.; et al. Securing the future of German manufacturing industry: recommendations for implementing the strategic initiative industrie 4.0: final report of the industrie 4.0 working group. Acatech. Alemanha, 2013.

[3] Nascimento, L. O. Fatores que influenciam a flexibilidade da produção de veículos comerciais. Dissertação de Mestrado, Universidade Estadual Paulista, Faculdade de Guaratinguetá, 2016.

[4] Muniz Jr., J. Panorama Geral da Organização Industrial, Univesp. Disponível em: https://www.youtube.com/watch?v=SBwg56ha81k Acesso em 20/11/2018

[5] Confederação Nacional Da Indústria. Indústria 4.0: novo desafio para as empresas brasileiras. 2016. Disponível em:< http://www.portaldaindustria.com.br/relacoesdotrabalho/media/pu blicacao/chamadas/SondEspecial_Industria4.0_Abril2016.pdf> Acesso em: 09/11/2018

[6] Cheng, Guo-Jian et al. Industry 4.0 Development and Application of Intelligent Manufacturing. 2016 International Conference on Information System and Artificial Intelligence (ISAI), Hong Kong, p.407-410, jun. 2016. IEEE. https://doi.org/ 10.1109/ISAI.2016.0092. Disponível
http://ieeexplore.ieee.org/document/7816745>.

Acesso em: 24 out. 2018

[7] Atzori, Luigi; IERA, Antonio; Morabito, Giacomo. The Internet of Things: A survey. Computer Networks, [s.1.], v. 54, n. 15, p.2787-2805, out. 2010. Elsevier BV. http://dx.doi.org/10.1016/j.comnet.2010.05.010. Disponível em: <http://www.sciencedirect.com/science/article/pii/S13891286100 01568>. Acesso em: 05 set. 2018.

[8] Bosch Software Innovations GMBH. Rule-based analysis of production data - manufacturing experts at the heart of Industry 4.0. 2017. Disponível em: <https://www.boschsi.com/manufacturing/insights/downloads/rule-basedanalysis.html>. Acesso em: 24 abr. 2017.

[9] Gaia, P. A quarta revolução industrial e as tendências tecnológicas no segmento de equipamentos, máquinas e acessórios industriais. O Papel: revista mensal de tecnologia em celulose e papel, v. 77, n. 5, p. 21-25, 2016. Disponível 
em:https://www.celuloseonline.com.br/47807-2/ >. Acesso em: 19/03/2018.

[10] Volkswagen Do Brasil, 2017, p. 4

[11] Griesinger, D. Pesquisa revela perfil da indústria 4.0 no Brasil. Disponível em: <http://agenciabrasil.ebc.com.br/pesquisa-einovacao/noticia/2016-5/pesquisa-revela-perfil-da-industria-40no-brasil>. Acessado em 17/11/2017.

[12] World Economic Forum. The Global Competitiveness Report

Disponível

em: <https://www.weforum.org/reports/the-globalcompetitivenessreport-2017-2018>. Acessado em: 05/10/2018.

[13] Heidrich, F.; Facó, J. F. B.; Reis, C. F. B. O impacto competitivo na indústria brasileira com a aplicação dos conceitos competitivos da Indústria 4.0. Anais do SIMPOI 2017, São Paulo, 2017

Disponível

em:

https://www.researchgate.net/publication/318403016_O_IMPAC TO_COMPETITIVO_NA_INDUSTRIA_BRASILEIRA_COM_ A_APLICACAO_DOS_CONCEITOS_DA_INDUSTRIA_40>. Acesso em: 29/08/2018.

[14] Accenture Strategy. The growth game-changer: how the industrial internet of things can drive progress and prosperity. 2015. Disponível em: <https://www.accenture.com/_acnmedia/Accenture/ConversionAs sets/DotCom/Documents/G lobal/PDF/Dualpub_18/AccentureExecutiveSummary-Growth-Game-Changer-Industrial-

Internet.pdf\#zoom=50>. Acesso em: 09/03/2018.

[15] McKinsey Digital. Industry 4.0: how to navigate digitization of the manufacturing sector. Disponível em: $<$ https://www.mckinsey.com/ business-functions/operations/ourinsights/industry-four-point-o-howto-navigae-the-digitization-ofthe-manufacturing-sector>. Acessado em 15/10/2017. 